\title{
How Technological Platforms Reconfigure Science-Industry Relations: The Case of Micro- and Nanotechnology
}

\author{
Martina Merz • Peter Biniok
}

Published online: 26 May 2010

(C) Springer Science+Business Media B.V. 2010

\begin{abstract}
With reference to the recent science studies debate on the nature of science-industry relationship, this article focuses on a novel organizational form: the technological platform. Considering the field of micro- and nanotechnology in Switzerland, it investigates how technological platforms participate in framing science-industry activities. On the basis of a comparative analysis of three technological platforms, it shows that the platforms relate distinctly to academic and to industrial users. It distinguishes three pairs of user models, one model in each pair pertaining to how platforms act toward and conceive of academic users, the other model regarding users from industry. The article then discusses how technological platforms reconfigure the science-economy divide. While the observed platforms provide new institutional contact and interaction between academia and industry, new research collaboration does not necessarily materialize in practice. In this respect, science-industry mediation by way of technological platforms does not make science-industry boundaries more porous. Instead, the declared openness of public research with respect to industry, in the case of technological platforms, may contribute to maintain public science's autonomy.
\end{abstract}

Keywords Technological platforms · Science-industry relations ·

Micro- and nanotechnology · User models

\section{Introduction}

Many science studies scholars concur in the observation that the relation between academic science and industry has undergone significant modification over the last

M. Merz $(\bowtie) \cdot$ P. Biniok

Institute of Sociology, University of Lucerne, Bruchstrasse 43, PO Box 7456, 6000 Lucerne 7,

Switzerland

e-mail: martina.merz@unilu.ch 
decades, with universities and public research organizations establishing dedicated organizational units, programs, and frameworks to foster science-industry activity. ${ }^{1}$ However, scholars disagree about how to conceptually handle this shifting scienceindustry link. Proponents of the "new production of knowledge" thesis (Gibbons et al. 1994) conceive of the dynamics as one of de-differentiation. In their view the demarcation between science and non-science has started to break down with the result that the autonomous space of science is no longer guaranteed (Nowotny et al. 2001). Other authors argue for the enduring existence of the boundaries of science and maintain that the observed mechanisms of mediation between academic science and industry rather reinforce than dissolve these boundaries. According to Weingart (2001, Chap. 5), for example, the relation of university and industry is characterized by a "closer coupling" (ibid. 176) today, which emphasizes the boundary's significance and turns it into a matter of reciprocal conflict and reflection. Shinn and Lamy (2006) similarly suggest the existence of a "highly complex positive correlation between the degree of university-enterprise synergy and degree of academic autonomy" (ibid. 1466). ${ }^{2}$

This seeming paradox between increasing contact and interrelation on the one hand, and maintained academic autonomy on the other, has been insufficiently explored empirically so far. The present article takes its inspiration from the apparent paradox to investigate science-industry interaction and dynamics with a focus on a specific organizational form: the technological platform. The emphasis will be on the complex interrelation between academia and industry and the multiple forms it can assume. The article will be attentive also to the reconfigurations occurring within academic science in association with the reconfigured academia-industry relations.

Academia-industry relations have been addressed in the science studies literature predominantly with a focus on knowledge transfer from academic science to industry by way of either persons or patents. This brings into view academic spinoffs, university patenting regimes, technology transfer modes, and the likephenomena which have given rise to a rich body of literature. ${ }^{3}$ In contrast, the interest and practice of firms to directly access academic science's experimental resources (expertise, skills, instrumentation) has received considerably less attention. ${ }^{4}$ Technological platforms at universities and public research institutions are of special interest in this regard. These instrumental facilities typically serve both academic and industrial users. They supply enterprises with access to academic

\footnotetext{
${ }^{1}$ Cf. e.g. Carayol (2003), Etzkowitz and Leydesdorff (2000), Mirowski and Sent (2008), Nowotny et al. (2001), Owen-Smith (2003), Shinn (1998), Slaughter and Leslie (1997), and Weingart (2001).

2 Shinn and Lamy (2006) discuss how scientists who have created their own company sit between and connect with the worlds of academia and business, showing that a maximum of university-enterprise synergy can coincide with an elevated autonomy of the scientific field.

3 Cf. e.g. Bozeman (2000), Meyer-Krahmer and Schmoch (1998), Owen-Smith (2005), Potthast and Guggenheim (2008), Tuunainen and Knuuttila (2009).

${ }^{4}$ But see Faulkner and Senker (1994) and, as a follow-up, Rappert et al. (1999) for a qualitative investigation of the public-private sector research linkage that identifies the benefits of firms also in terms of "assistance with experimentation" such as skills in new techniques, access to research equipment, and research materials.
} 
research infrastructure and, simultaneously, afford novel formats of on-site contact and cooperation with academic science. Especially in the realm of micro- and nanotechnology, technological platforms have been promoted with the promise that they foster new forms of academia-industry cooperation and partnership, the intensified ties being seen as constitutive for the emergence and consolidation of these research fields (cf. Hubert 2009; Peerbaye and Mangematin 2005; Robinson et al. 2007, see also below).

The present article will look into these issues for the case of Swiss publicly funded research. During the last ten years, Swiss universities and Federal Institutes of Technology have established a number of "platforms," "facilities," and "service labs" that target research in micro- and nanotechnology. These units provide scientific and technological equipment and expertise (various forms of microscopy, specimen preparation, micro- and nanolithography, characterization, etc.), the corresponding personnel, and dedicated work environments (e.g. clean rooms). How can these facilities be described and what forms of science-industry interaction do they promote in discourse and practice?

This article investigates the relation between academic science and industry from the perspective of the technological platform, i.e. its staff. The motivation for this choice is an interest in how this new organizational form participates in framing science-industry activities. The analysis is guided by a twofold hypothesis. It maintains that, first, technological platforms differ in how they relate to and conceive of their respective users. Second, a technological platform treats and views academic and industrial users distinctly—i.e. it establishes a pair of "user models," one model pertaining to academic users, the other to industrial users. In line with this hypothesis, the aim of the article is to identify central aspects of the technological platforms' distinct user models and discuss them in the context of the current debate on the shifting science-industry link. To our knowledge, none of the still infrequent studies of technological platforms share this preoccupation.

The platforms' user relations have an organizational and a symbolic dimension. The organizational dimension concerns how platforms set up and realize, in practice, the contact and exchange with users; the symbolic dimension refers to how the platform staff views users and how it conceives of the platform's position and role with respect to users and the institutional environment more generally. Both dimensions are intimately connected but need not coincide.

Our interest in technological platforms and the associated user relations has developed as part of a more extended project, in which we analyze the configuration of nanoscale research in Swiss publicly funded science (see Acknowledgments). A combination of complementary qualitative research methods has produced a comprehensive data set, a fraction of which is expedient to the current investigation into technological platforms and their user models. Documents such as a platform's annual reports, conference presentations, and user manuals provide data concerning its organizational (competences, instrumentation, procedures, etc.) and symbolic (programmatic statements, visions, rationales, etc.) dimensions. Altogether, fifteen expert interviews with scientific staff and selected users inform about the platform organization as well as the conceptions of staff-user relations. The documents and 
interview transcripts were supplemented by notes from occasional participant observation of the daily activities at the considered technological platforms. ${ }^{5}$

The article first introduces prevailing notions of technological platforms and spells out the underlying rationales. It then portrays three technological platforms in micro- and nanotechnology that form the empirical basis of the current investigation. The analysis of how platforms perceive their users results in the identification of distinct user models, which are presented consecutively. Finally, the results are discussed and contextualized.

\section{Technological Platforms: Central Concepts}

The notion "technological platform" 6 refers to facilities in a range of scientific, medical and technological areas and has also become a concept in a number of social sciences. In their fine book on Biomedical Platforms, science studies scholars Keating and Cambrosio (2003) provide an etymology of the term "platform" as well as a review of the recent literature. In the historical development of how the term is used, they identify "a shift in connotation from platforms as passive supports to platforms as springboards for future action" (ibid. 27). While less interested in the term's changing undertones across time, we observe that also recent notions of (technological) platform sit in between the two poles of a more passive ("support") and a more active ("springboard") interpretation of their effects and workings, as the following examples illustrate.

In computer science, a platform is viewed as a technological (software and hardware) basis for computer, information, and communication systems; this understanding emphasizes above all the platform's materiality. From an economic perspective, capabilities are considered as technological platforms when they open up opportunities to participate in future markets, an association which recalls the "springboard" notion of platforms (e.g. Kim and Kogut 1996). Organization studies envision platforms as a means of engendering new arrangements of resources, routines, and structures, which also corresponds to a more active reading (e.g. Ciborra 1996; Gerstein 1992). Extending the concept to that of "platform organization," Ciborra (1996), for example, views a platform as "a formative context that molds structures" (ibid. 103) and that is "suited to cope with chaotic environments" (ibid. 113).

In the field of science studies, the discussion of technological platforms similar to the ones we observed has only just started. As a consequence, a coherent analytical concept of "technological platform" is still lacking. This contrasts with research on "biomedical platforms" where notably the work of Keating and Cambrosio (2000, 2003) has been seminal in promoting a coherent, more abstract concept of platform. The two authors define biomedical platforms as specific configurations of the biomedical process, set apart e.g. from infrastructure and experimental systems.

\footnotetext{
${ }^{5}$ However, due to its preference for the perspective of the platform staff, the article is less interested in providing a "thick description" (Geertz 1973) of the users' experiences and practices.

6 Some authors use "technology platform" instead of "technological platform" but we could not identify a systematic difference between the two notions.
} 
In contrast to infrastructure, these platforms are "active, generative, and opaque"; in contrast to Rheinberger's notion of experimental systems, the focus is not on the production of epistemic things but on their regulation (2003: 326-328). Such platforms are seen "as material and discursive arrangements that act as the bench upon which conventions concerning the biological or normal are connected with conventions concerning the medical or pathological" (2003: 4).

At the same time, another set of science studies investigations has adopted a notion of technological platform similar to the notion of research facility. ${ }^{7}$ A first example is the study of Peerbaye and Mangematin (2005) who define technological platforms "as research and/or production facilities required to explore and exploit new knowledge," characterizing them further as "complex assemblages of instruments and expertise" and emphasizing that they "are more than often 'cross-boundary' devices, whether geographic, scientific, or organisational" (ibid. 28). With a focus on the life sciences, the authors analyze the sharing of infrastructure as a technology transfer mechanism from public sector research to industry, identifying three organizational models (academic, private, public/private) according to their specific effects on industrial dynamics.

A second study similarly defines a technological platform as "a set of instruments which enables scientific and technological production" while at the same time highlighting that it "is not just a collection of equipment" but also "enables and constrains further actions" (Robinson et al. 2007: 872). In their investigation of two extended nanotechnology clusters in France and the Netherlands, the authors adopt a springboard notion of platforms when they argue that the co-location of scientific and technological fields and the existence of shared technology platforms decisively foster the emergence of nanotechnology clusters.

In a third case, Hubert (2009) discusses technological platforms, here considered as a synonym of "user facilities," in a study of a major French nanotechnology cluster under the notion of an "externalization of experimentation beyond the laboratory." 8 This study involves an exploration of how the relation of laboratory and experimentation is reconfigured through the outsourcing of instrumentation to the platform with an interest in organizational models of experimental activities.

In summary, we identify two prevalent platform notions in present science studies scholarship: "biomedical platforms" as conceptualized by Keating and Cambrosio and technological platforms interpreted as research facilities. The distinction between the two can be highlighted by the observation that a biomedical platform is not an organizational unit and can exist in any type of laboratory, whereas a technological platform as research facility typically coincides with an organizational unit. Yet, despite their distinct conceptual orientations and empirical bases, it might be productive to contrast the two perspectives to incite topics for future research. ${ }^{9}$

\footnotetext{
7 For a distinct notion of technological platform as "foundational technology," see Lenoir and Giannella (2010).

8 Our translation from the original French: "externalisation de l'expérimentation hors du laboratoire" (ibid. 23).

${ }^{9}$ For example, it remains a challenging question how platforms are associated differentially with epistemic cultures, be it in the interpretation of Keating and Cambrosio, or be it in the alternative one.
} 
This article takes its inspiration from both bodies of work. We will reflect on platforms as socio-technical arrangements that associate heterogeneous entities to enable scientific work: scientists, engineers and technicians as platform staff; a local environment that affords face-to-face interaction between people and people's direct access to equipment; an assemblage of instrumentation, techniques, skills, and infrastructure; users of different disciplinary and institutional background and varying career stages; etc.

\section{Technological Platforms in Micro- and Nanotechnology}

In micro- and nanotechnology, technological platforms are a recent phenomenon. The two fields involve scientists from a broad range of disciplines, such as physics, chemistry, life sciences, materials science, and other engineering sciences. Some platforms with their instrumentation are exclusive to these domains (e.g. clean room facilities) while others are of interest also to researchers of other orientation (e.g. microscopy centers). For this reason, the technological platforms that are central to micro- and nanotechnology vary significantly with respect to the exclusivity of the offered service, the spectrum of users, and their position in the respective university or research institute.

The rationales for the technological platforms' constitution are presented as a package. Typically, a first advantage put forward by platform staff concerns the elevated cost of instrumentation in the field, seen as an incentive to share it among numerous users. While some instrumentation is indeed exclusive to technological platforms (e.g. a synchrotron facility or robots to process wafers), other instruments are present in smaller laboratories as well as in platforms (e.g. probe and electron microscopes). In addition to the claim that costs are saved when instrumentation is centralized and pooled, a second advantage put forward is that technological platforms centralize expertise, maintenance, support, and training. This combination of care for the apparatus and care for the users is more difficult to provide in the context of smaller laboratories. The capacity to maintain these activities over extended periods of time and therewith provide for continuity is a third benefit promoted by platforms.

The technological platforms selected for empirical investigation are part of public institutions of research and higher education in Switzerland, and they offer their services to both academic researchers and to industrial partners. Whether they prioritize academic users, treat all users alike or specifically approach industrial users varies from one platform to another. The observed variation in how technological platforms conceive of and relate to their users has led us to propose the aforementioned double hypothesis: that platforms have dissimilar user conceptions and relations, and that they typically view and handle academic and industrial users distinctly. In line with this hypothesis, we have identified the user models of each technological platform. In principle, it is of course conceivable that a technological platform establishes more than two user models, fanning out its user categories in a more fine-grained manner. In practice, however, we have observed that the main dividing line is the one between academic and industrial users, 
Table 1 Technological platforms in comparison

\begin{tabular}{|c|c|c|c|}
\hline & $\begin{array}{l}\text { Center of MicroNano } \\
\text { Technology/CMI }\end{array}$ & Swiss Light Source/SLS & $\begin{array}{l}\text { Swiss Scanning Probe } \\
\text { Microscopy User } \\
\text { Laboratory/SUL }\end{array}$ \\
\hline $\begin{array}{l}\text { Start of } \\
\text { operation }\end{array}$ & 1999 & 2001 & 2006 \\
\hline Affiliation & $\begin{array}{l}\text { EPFL (Ecole } \\
\text { Polytechnique Fédérale } \\
\text { de Lausanne) }\end{array}$ & $\begin{array}{l}\text { PSI (Paul Scherrer } \\
\text { Institute) }\end{array}$ & $\begin{array}{l}\text { Empa (Swiss federal } \\
\text { laboratories for materials } \\
\text { testing and research) }\end{array}$ \\
\hline Type of lab & User lab & User lab & Service \& user lab \\
\hline Instrumentation & Clean room facility & Synchrotron light source & Atomic force microscopy \\
\hline $\begin{array}{l}\text { Academic } \\
\text { users }\end{array}$ & $\begin{array}{l}\text { Life sciences, electronics, } \\
\text { quantum optics } \\
\text { Mostly EPFL }\end{array}$ & $\begin{array}{l}\text { Physics \& materials } \\
\text { science, structural } \\
\text { biology, chemistry } \\
\text { Swiss \& international }\end{array}$ & $\begin{array}{l}\text { So far, only a few scattered } \\
\text { users }\end{array}$ \\
\hline Industrial users & $\begin{array}{l}\text { Small local companies, } \\
\text { start-ups, watch- } \\
\text { making industry }\end{array}$ & $\begin{array}{l}\text { Variety of firms, } \\
\text { pharmaceutical industry } \\
\text { and ICT }\end{array}$ & $\begin{array}{l}\text { Local industry, mostly } \\
\text { chemical industry }\end{array}$ \\
\hline
\end{tabular}

providing for pairs of user models. We will advance the thesis that the models in a pair are not independent but interrelated (cf. "Conclusions").

In this article, three exemplary cases of technological platforms that operate in micro- and nanotechnology research are discussed. While the platforms share the typical characteristics presented above, they differ in many other respects, concerning the type, size and cost of instrumentation, their age and maturity, the disciplinary and organizational background of their users, etc. (cf. Table 1). The choice of the three platforms is motivated by the fact that they allow us to present three distinct pairs of user models. For a particular technological platform, we have accentuated the features that characterize the specific user models. In this sense, the models can be understood also as ideal types. While we have looked into the case of additional platforms in Swiss micro- and nanotechnology research, we could not identify any substantially different user models. Nonetheless, we do not want to go as far as claiming that only the three identified pairs exist. In the next three sections each of the platforms will be discussed in turn.

\section{A Micro- and Nanotechnology Clean Room Facility}

The Center of MicroNanoTechnology (CMI) is a central clean room facility at the Swiss Federal Institute of Technology in Lausanne EPFL (Ecole Polytechnique Fédérale de Lausanne) and started operation in 1999. Its objective is to provide access to research equipment for scientists, above all from the own institution, the EPFL, but also to external academic and industrial users.

The staff of CMI is composed of the director, a professor at the Microsystems Laboratory of EPFL, and the director of operations who is responsible for the dayto-day running of the facility. In addition, the staff comprises four "section heads" 
(dedicated sections on photolithography, etching, thin films, nanotools) with academic credentials and a number of engineers and technicians, altogether about twenty people. About half of the staff is on permanent positions from EPFL to assure the continuity of expertise and maintenance and to gather a knowledge pool that does not migrate.

A section head describes the set-up of the CMI as following a "semi-industrial model," referring to the typical operational procedures, the type of technological infrastructure and the spatial distribution of the work processes. The CMI is divided into different zones that are dedicated to different processes and equipment, such as photolithography, wet and dry etching or nanotools. The users process full wafers, which are central components of microdevices such as integrated circuits. Wafers undergo many process steps, which are handled at dedicated benches, each bench being set up to do one precise process.

The CMI also provides automated machinery and robotic handling, which is unusual for a university facility. As a result, individual tasks are strongly guided and there is little to no flexibility to adapt them. At the CMI "there is no play area" (as a staff member put it), which distinguishes its operation from a more traditional academic lab. A $\mathrm{PhD}$ student, academic user of CMI, provides an example. A vapor deposition machine is available both at the CMI and in his supervisor's lab but in contrast to the CMI machine, which runs with predetermined materials, the team machine is more flexible and allows the scientists to experiment with different materials.

In line with the type of technology, a few staff members have an industrial background. All equipment was freshly purchased for inclusion in the CMI. It provides novel equipment and expertise that were not accessible to EPFL scientists previously.

Typically, the staff assists the scientists to use the apparatus by themselves: CMI is a user lab. Exceptions may be granted, when the staff expects that training a scientist will be more time-consuming than to provide the service. If staff members provide assistance, they monitor the work process very closely. They wait until results are obtained and check them together with the users. In many cases it was difficult for us, as observers, to distinguish staff-user cooperation from user-user collaboration. Besides the fact that all people wear the same protective clothing, be they staff members, industrial or academic users, the communication is informal and both staff and users handle the apparatus.

The first model pair, exemplified by the CMI, opposes an educational model with respect to academic users and an indifference model with respect to industrial users.

\section{Educational Model}

The academic users are mainly composed of $\mathrm{PhD}$ students and postdoctoral researchers. The specific feature of this model is that the platform staff engages in a relation of protective care with each user individually, guiding him or her through the respective project. In a first step, before entering the clean room, the future user has to write up the concrete project with the envisaged procedures for work in the clean room. The platform staff (section heads, engineers and technicians) evaluates 
this proposal in a face-to-face meeting with the scientist, the so-called "technical committee." The staff thus interferes right at the beginning of a project to make it "workable," which may entail a radical modification of the original plan. After the project has been accepted, the user follows an introductory seminar on safety rules and clean room guidelines. Before the actual work starts, the user also receives detailed instruction. The CMI has no global training policy but trains each scientist individually in dedicated training sessions that are scheduled according to a project's requirements. In the words of a CMI section head:

"People are trained individually. (...) It's the heart of our job if you like. It's to train people on equipment" (Section head at CMI, interview).

The technological platform's staff thus assumes the role of supervisor and tutor. The strong guidance by the personnel emphasizes the educational undertaking of CMI. As users become more experienced, the staff gently withdraws. But staff members are always available on-call for support. A postdoctoral user explains to whom he will turn for support:

"Usually [I ask] the responsible from CMI. There are always about a few people responsible for the same machine, so I think they make pretty sure that somebody is available from 8 to 5 to help you out" (Academic user, interview).

At CMI, academic scientists are not only educated individually. The environment with its spatial organization-different benches positioned in close vicinity, scientists moving frequently from one bench to another, crossing the open space-encourages contact and interaction (Gieryn 2002). Beginners consult more experienced users for advice. Hints are shared across the open space. The staff is available for all other problems.

\section{Indifference Model}

With respect to industrial users, the technological platform follows what we would like to call an indifference model. This does not imply that these users are not welcome, quite the contrary: Industrial activity at CMI amounts to about $20 \%$ of all projects, mainly by small companies and start-ups (according to statistics provided by CMI in 2008). Entry to the lab is regulated by priority rules, granting first priority to EPFL researchers over external academic users and industrial research. However, as of yet, CMI has managed to re-channel access to instrumentation in such a way that no potential user had to be turned down, which holds also for industrial users. They are charged by the hour for work in the clean room with fees that are not substantially higher than those paid by academic users.

The technological platform is indifferent to industrial users in the sense that it does not accommodate their special needs. As concerns the situation of industrial users, all service and training options are open to them in principle. They may undergo a technical committee if they wish (but typically prefer not to disclose their project's detailed procedures) and they are trained according to their preferences. 
But equal treatment also implies that no effort is undertaken to accommodate industry's preferences and specific needs. For example, the secrecy of projects, procedures and results is not warranted. This refers to the general aims of the project as well as to measurement results. A CMI staff frames this lack of accommodation in terms of the (non-monetary) price companies have to pay for using the clean room.

"The price you pay for using our lab is that you tell us what you are doing" (Section head at CMI, interview).

And, concerning the fact that scanning electron microscopy images that are produced on one of the CMI instruments will be visible to other users as well:

"If industrial users don't want their images remaining on the public server, then it's up to them to take them off. But it's not something we are going to take care of, this is again the price they pay for coming to use our labs" (Section head at CMI, interview).

Both statements refer to CMI being part of a publicly funded academic institution. The technological platform is indifferent not to industrial users per se but to a company's potential special needs, such as issues of secrecy and intellectual property protection. As long as they go along with the academic practices that prevail at CMI, industrial users are welcome. Should they not be willing to adapt to this mode of work, a CMI staff member suggests that they "build their own lab."

Interestingly, this attitude does not seem to cause unease among industrial users, at least not to those with whom we spoke. They are comfortable to work in the open space of the facility because the specific characteristics of wafer processing (i.e. the component they feel secretive about) can remain concealed. They also readily accept that in-house users have priority to use the machines. They view the CMI as an essential technology provider that allows them to develop and test technologies. CMI being a user lab enables industrial users to gain hand-on experience, a feature that may increase their credibility with respect to their customers. In the words of an industrial user:

"And that is improving our credibility towards our customers and partners a lot. Because we're not a kind of fabless company, where people control around chips and say: 'That's what we can do but if you need to know how it was done, we have no idea.' We already know, down to the equipment levels, what temperature to set and what can be the sensitive part of it" (Industrial user, interview).

To conclude, due to its affiliation with an institute of higher education, the platform personnel is anxious to fulfill its academic and educational mission. To educate and train the next generation of academic researchers is their aim and contribution to society. This mission seems to extend also to certain industrial users who are treated in the same way as academic users in the clean room. Among the companies that use CMI are spin-offs founded by former doctoral students of EPFL who return to the clean room in their new positions and affiliations. The CMI staff receives them 
favorably as one of theirs; the educational role of the CMI thus extends to the offspring even when it has grown up.

\section{A Synchrotron Radiation Facility}

After a planning and building phase of about 10 years, the Swiss Light Source (SLS) started operation at the Paul Scherrer Institute (PSI) in 2001. The ETH Board and the Swiss Federal Council secured the financial support. The motivation behind the construction of the SLS was to provide Swiss scholars with their own synchrotron source. But the project also offered a new raison d'être to the PSI, which was looking for a new scientific orientation in the late 1980s.

The Swiss Light Source is a typical "big science" platform. ${ }^{10}$ It is extended in size (the storage ring has a circumference of $288 \mathrm{~m}$ ), it is costly, it requires a large number of people for maintenance and operation, etc. Three Laboratories for Synchrotron Radiation manage the activities at SLS and provide services. They cover a broad range of fields, including environmental and life sciences, condensed matter and materials sciences, and micro- and nanotechnology. The combined staff amounts to about fifty scientists and technicians each. The size of the machine, the number of beamlines and the fact that the machine runs 24 hours a day and 7 days a week accounts for this large number of staff.

The SLS is a source of synchrotron light, which is generated by high-energy electrons circulating for hours in a so-called storage ring. Synchrotron light is electromagnetic radiation with a spectrum that ranges from infrared light to X-rays and is sharply focused. For this reason it is a powerful instrument to probe surfaces as well as bulk properties of novel structures and materials. The SLS provides beamlines of different wavelength that guide the synchrotron light to experimental stations. In 2008, seventeen lines were in use and four additional ones in a planning or construction stage. The individual lines are used for different kinds of experiments (such as protein crystallography).

Each beamline area is made up of several cabins with computers and additional equipment where distinct user teams perform their experiments. While we described the SLS as a big science platform, the beamline teams and experiments are, in fact, rather small. Typically, each beamline is under care of a team of two scientists, a technician and one or two postdocs. The scientists and to some extent also the postdocs provide service in form of consultation and assistance. In addition, they are involved with "in-house research," i.e. they develop und upgrade the beamline technology. Staff members also follow their own research agenda and publish accordingly.

On its webpage, SLS describes itself as a user facility. More than half of the users work independently after receiving initial assistance; the others require more attention. Depending on the expertise of the users and the difficulty of the experiments, the beamline scientists invest more or less time to assist the users. In case many different measurements have to be carried out or the users need extended

\footnotetext{
${ }^{10}$ For the concept of big science, cf. Galison and Hevly (1992).
} 
assistance, the staff remains at the beamline, helps to adjust the apparatus and specifies the control parameters. In case users are experienced or measurements are repetitive, it suffices that the staff adjusts the beamline in the morning. The users conduct the experiments on their own and replace the sample when required. Users typically stay in their cabins where they run the experiments in shifts. Except for these cabins there are no dedicated areas where users can meet und talk-in contrast to the clean room facility CMI whose spatial setup encourages users to interact.

Besides researchers from Swiss institutions, international academics and industrial users work at the SLS as well. In the first years of its existence, the installation provided more capacity than required by national scholars, which created another incentive to attract users from abroad and from industry. Recently the number of experiments has increased sharply with the result that the capacity today is exhausted. According to the PSI 2007 Annual Report, "demand exceeds the supply by far."

The second model pair differs considerably from the combination of an educational model and an indifference model as witnessed at CMI. It consists of a competition model targeting academic users and a purchase model concerning industrial users.

\section{Competition Model}

The SLS is open to academic users from all countries free of charge. Swiss scholars have no priority. The motivation for the equal access policy is that comparable centers in other countries follow a similar policy. This alignment is judged favorably due to its promoting exchange and international cooperation. The procedure to regulate access is closely associated with the notion of scientific excellence. International competition is claimed to stimulate the quality of research. The technological platform's credo is: "If the scientific quality is right, you have access" (head of one of the SLS laboratories). In practice, scientists interested in using a beamline have to submit a research proposal, which is first checked for feasibility by the beamline scientists and then submitted to peer review by an external and independent international panel. The panel, consisting of five thematic committees that meet twice a year, provides a list of projects, ranking them according to scientific quality. On the basis of this list, the SLS staff allots beam time to the applicants. Since beam time is a sparse good (on the organizing principle of restricted beam time, cf. Traweek 1992), it is exploited fully and cautiously, measurements being conducted around the clock. The competition model thus guides the platform's relation with its users. But it has consequences also for the SLS staff in their role as researchers (most of them being involved in their own research projects), as they have to compete for beamline access with all other applicants and according to the same procedures.

The relationship between academic users and beamline scientists is not only one of support and competition, but also of collaboration. Different forms of collaboration can be distinguished, a first form consisting of shared responsibility for measurement and data analysis and resulting in co-authored publications: 
"Yes, there exist scientific collaborations. We collaborate with a few groups [of academic users] by sharing responsibility for the measurements. To some extent, we also jointly analyze the data. (...) In all of these cases, our partners are in charge of project management. Such projects result in good publications, occasionally in Science and Nature, which makes it attractive" (SLS staff, interview). ${ }^{11}$

On other occasions, the beamline scientists' contribution to the experiments is mentioned in the resulting publication's acknowledgments.

The competition model (SLS) contrasts with the educational model (CMI) in two respects. First, access is granted according to distinct criteria and to a different set of scientists: criteria of excellence and international competition contrast with criteria of need and a priority for the home institution's users. Second, scientific staff and academic users are related in distinct ways. While the staff is viewed a potential collaborator in the case of SLS, staff members are predominantly considered as supervisors and tutors by the users of CMI.

\section{Purchase Model}

The competition model's counterpart is the purchase model, which concerns industrial users: according to this model, access is not channeled through scientific competition with peers but can be purchased. The SLS provides two different modalities for this purpose. According to the standard variant, beam time at a specific beamline can be acquired. At present, about $10 \%$ of the beam time is sold to companies. In parallel, another modality exists, which has been negotiated e.g. with the pharmaceutical corporations Roche and Novartis. The two have co-financed ( $25 \%$ each) a beamline dedicated to application in protein crystallography. In return, $50 \%$ of the beam time is at their disposal.

In contrast to the indifference model observed at CMI, the purchase model comes with an explicit policy to respect and accommodate intellectual property issues of industrial users. Results can be proprietary and need not be published. Accommodating industry implies also that the Roche and Novartis teams can work with their own technical staff and computer systems and have the entire experimental area of "their" beamline to themselves. In addition, they do not need to inform the platform staff about the projects they are working on. According to the SLS staff, the companies only have to assert that they do not handle dangerous substances and comply with ethical standards. The spatial configuration of the workspace with its individual lockable cabins affords work in secrecy.

"No, no, no. We have absolutely no insight [into the industrial users' projects]. All they have to do is guarantee that their samples are no source of danger. They have to acknowledge this in written form. And they also have to dispose of the samples themselves. Besides that, no, well, these are Roche and Novartis, in this case we don't ask" (SLS staff, interview). ${ }^{12}$

\footnotetext{
11 Our translation.

12 Our translation.
} 
To manage the purchase of beam time at PSI, a dedicated institution was founded in 1997: the SLS-Techno Trans AG. This service provider sells beam time together with a range of services (such as analyses and further investigation) to industrial users. Potential users interested in purchasing beam time have to contact and go through SLS-TT AG. Surprisingly, the purchase model is of interest not only to industrial users, but also to academic scientists who can buy beam time subject to the same conditions. Typically, these researchers have different incentives to select this modality, one being that they want to explore a new topic without needing to disclose the underlying ideas to potential competitors in the review process.

The realization of the purchase model is a result of the explicit interest of SLS (and the Paul Scherrer Institute, more generally) to attract industrial users to the platform. The participation of industry is important amongst others because it demonstrates and symbolizes the institute's reorientation toward applied science, thus distancing itself from the earlier dominance of fundamental research in a time when the PSI was strongly associated and involved with particle physics.

In contrast to the case of CMI with its dominant image of academic users, the SLS provides for a more symmetric relation: both types of users are equally welcome and both are accommodated with respect to their specific needs. Where academic users acquire beam time by demonstrating their scientific excellence in a competition, industrial users acquire beam time against money.

\section{A Scanning Probe Microscopy User Laboratory}

The third technological platform is a recent facility still in the process of establishing itself. In 2006, the Swiss federal laboratories for materials testing and research, Empa, in cooperation with the Competence Center for Materials Science and Technology (CCMX), set up the Swiss Scanning Probe Microscopy User Laboratory (SUL). Empa is one of the research institutes of the ETH Domain, which today comprises the two Swiss Federal Institutes of Technology (ETH Zurich and EPF Lausanne) and three other federal research institutions. In the last decade, Empa has undergone a major reorganization, in the course of which it has established a dedicated nanotechnology research program (cf. Merz 2010). SUL is an initiative associated with this program and, as such, part of the section "Nanoscale Materials Science."

The technological platform is devoted to materials (surface) analyses at the nanoscale. It provides analytical services and access to equipment such as a number of scanning probe microscopes (especially atomic force microscopes). The original motivation behind SUL was to provide a central infrastructure for Empa researchers instead of each department acquiring and maintaining their own isolated equipment. A second important motivation of the founders was to provide instrumentation for punctual utilization to institutions and companies that do not perform such measurements on a regular basis.

SUL is not only young; it is also (still) small. The prime contact person is a senior researcher who, next to her regular research activities, devotes one day a week to SUL. She is responsible for communicating with the users. Two other people are in 
charge of maintenance, service measurements and training, being employed parttime to work at SUL. The equipment at SUL, all of which was bought new, consists of five atomic force microscopes (AFM), among them standard instruments as well as specialized ones that can be used to measure magnetic samples or in extreme environments.

The staff refers to users as "clients" or "customers." This indicates the orientation of the lab as a service lab. Empa describes the SUL explicitly as a "service lab for materials analysis on the nanoscale" and promotes it with a reference to its history and service tradition:

"With the SUL we extend one of Empa's crucial traditional roles-namely to provide highest-level services and training to industrial and academic partners in the field of nanotechnology" (Hug 2007).

As a service lab, SUL performs measurements for interested parties unlike the two previously introduced technological platforms, which are user labs. This orientation corresponds to Empa's tradition as a materials testing institute.

\section{Service Model}

In contrast to the two other technological platforms, the scanning probe microscopy user lab SUL does not allow us to spell out the third model "pair," as the model for academic users is still so vague (due to the platform's young age) that its features do not emerge as a distinctive model (yet). In a sense, the situation is reverse compared to that of the discussed clean room facility. While the CMI had established a clear model of viewing and treating academic users, the SUL has an equally established model for how to deal with industrial users. The reason for this focus on external users (and especially those from industry) lies in the organizational culture of the Empa and its history.

Empa was founded in the late 19th century as an institute for testing materials. Since the late 1980s, research was upgraded with the result that Empa has today turned into a modern institute of materials research, which has considerably reduced (albeit not eliminated) its testing activities. Throughout its history, it has cooperated in research and development with industry, universities, and polytechnics; its testing assignments are commissioned by industry and public administration. Still today, it positions itself strategically at the interface of industry and science (cf. Merz 2010). This trajectory has consequences for the SUL. As a service laboratory it can draw on a long and rich experience of how to formalize, set up and manage service relations with external partners. At Empa — and thus also SUL-many features of service relations with industry are standardized. For example, there is an established practice of setting up service contracts (standard contract forms, fixed prices, etc.). The platform coordinator replies to our question of how SUL handles the issue of intellectual property rights:

"Well, this is common practice, we provide a lot of service here. It is, the data belong to the company, the contracting body, but the know-how, should we 
have learnt something about the instruments by way of the process, this stays with us" (SUL staff, interview). ${ }^{13}$

Thus, SUL can take advantage of established modes of handling issues of intellectual property rights. Specific secrecy contracts are available as well, although they are only rarely applied. In most cases, personnel at SUL has little specific knowledge of the projects underlying the contracts and is confined to the measurement tasks.

At this early stage in the life of SUL, its staff are active on several fronts at once with the following aims: To convince companies that atomic force microscopes are not merely a research tool but can be usefully applied for the purpose of industry; to work toward the declared aim of fostering cooperation between Empa scientists and industry by way of the SUL service model; to attract academic users to SUL; to train potential users and provide equipment instead of providing service in the form of measurements - i.e. to open up a venue toward a user model. However, at the moment the service model seems solid, as it ties in with organizational practice and professional culture at the same time.

\section{Conclusions}

By way of technological platforms a novel format of academia-industry interaction has surfaced at universities and public research organizations. Technological platforms centralize, pool, and maintain instrumentation and provide users with access to equipment, with advice, assistance, and training. The observed platforms target academic and industrial users conjointly, but they have established dedicated conditions of usage, treating representatives of academia and industry according to dissimilar user models.

In the introduction we asked how technological platforms, as a new organizational form, participate in framing science-industry activities. We raised this question in the context of a purported paradox: that increased academia-industry contact can coincide with the maintenance of academic autonomy. How do the above observations relate to this seeming paradox? On the one hand, technological platforms do indeed provide new means to increase contact and interrelation between academic science and industry. Interrelation here refers first of all to the provision of new means for industrial actors to access academic infrastructure. On the other hand, this does not necessarily entail close contact and cooperation between academic researchers and industrial users. The exploitation of the same set of instrumental resources does not automatically engender close affinity and cooperation among different users, especially if they do not share conditions of usage due to dissimilar user models. Furthermore, we did not observe any specific policies to encourage academia-industry research cooperation mediated by the technological platforms. Quite on the contrary, certain platforms attract industrial users by providing ways for them to protect their activities from the eyes of

13 Our translation. 
academic users and therewith minimize occasions to establish contact. Academic and industrial modes of access seem to proceed in parallel, without negative interference but also without observable cross-fertilization.

When looked at closely on the basis of these observations, i.e. also by considering the difference between de-differentiation and demarcation not as theoretical assertion but as practical accomplishment, the purported paradox dissolves. This dissolution occurs once it is realized that academia and industry are no homogenous entities as regards platforms. On the side of public science, researchers and representatives of technological platforms relate to industrial actors differently. Thus, while technological platforms may provide new institutional contact and interaction between academia and industry, new research collaboration does not necessarily materialize in practice. In this respect, science-industry mediation through technological platforms does not make science-industry boundaries more porous. Instead, the declared openness of public research with respect to industry, in the case of technological platforms, may contribute to maintain public science's autonomy.

These observations also relate to the concept of technological platform that is appropriate. As discussed, social scientists oscillate between two notions of platform: platform as passive support and as springboard for future action. In our view it is misleading to posit a springboard notion of technological platform from the outset because it may seduce analysts to privilege an "organizationally determinist" (i.e. in analogy to a technically determinist) vision of platforms. Instead, we conceive of platforms as situated differentially in the spectrum spanned by "passive support" as one pole and "springboard for future action" as the other, with respect to specific usage situations and forms. For example, it is conceivable that some platforms (such as CMI at EPFL) provide mere passive support to industrial users while opening up new ways of research cooperation for academic users. This question cannot be answered in the context of this article, as it would require a more detailed investigation of user practices.

As discussed above, the considered technological platforms exhibit different (pairs of) user models. We suggest that this variation is associated with the diverse organizational cultures of the platforms' mother organizations: a university, a highprofile research lab, and a former service lab. Let us reconsider each case separately.

- The Center of MicroNanoTechnology (CMI) being an organizational unit of the technical university EPFL, it does not come as a surprise that it relates to its users corresponding to a university culture. The platform staff strongly identifies with academic education as its mission, offering training and supervision to users, among which are many junior researchers at the EPFL. By treating industrial users like their academic peers, the CMI staff extends the educational gesture. Yet, at the same time the similar treatment implies that the industrial actors' specific needs are not accounted for: the platform staff exhibits indifference. This indifference model is manifestly subordinate to the educational model and academic autonomy is clearly maintained.

- The Swiss Light Source (SLS) is a central facility of the internationally esteemed research center PSI and shares the tenets of its research culture. The 
platform staff emphasizes its objective to enable outstanding research by selecting academic users according to criteria of excellence in a competition model. How does this correspond with the purchase model adopted with regard to industrial users? It seems (but more research is needed to provide a conclusive answer) that the coupling of a competition and a purchase model can be explained by PSI's recent organizational history, especially its reorientation toward applied science and technology transfer. Providing for two distinct modes of operation (and user models) — one privileging the currency of scientific excellence, the other the one of monetary compensation-allows fundamental research to coexist besides industrial application without interference. This model pair most closely illustrates how academic autonomy can prevail simultaneously with public research-enterprise synergy.

- The mother organization of the Swiss Scanning Probe Microscopy Lab (SUL), Empa, provides an example for an organization whose evolution has moved in the opposite direction: Traditionally identified as a testing laboratory with a predominant service orientation, Empa has recently upgraded its research competencies. It is expected to walk a fine line combining state of the art research and the provision of high-level service. The service model established by SUL meets these requirements. It primarily targets industrial users, drawing on established procedures to handle this form of private-public cooperation. In contrast to the two other considered technological platforms with their established models of handling academic users, the SUL is still in the process of devising such a model.

The above observations suggest that the coupling of a specific "academic user model" and a particular "industrial user model" is not arbitrary. Yet, different sets of combination seem possible, depending on a specific organization's culture and a set of contingencies, related to a technological platform's and its mother organization's history.

Last but not least, we are aware that the above observations might be specific to the case of Switzerland. It is possible that the observed platforms' initiatives to cater to industry are more "hesitant" in this country than in others. Especially in the area of nanotechnology, the constitution of technological platforms has come to symbolize intensified academia-industry partnership and cooperation in certain other national contexts (e.g. in the cases of France and the Netherlands, cf. Robinson et al. 2007). In Switzerland, a joint public-private platform project of ETH Zurich and IBM Switzerland is currently under construction. But it is too early to tell whether new user models will emerge from this endeavor with more intimate research cooperation between academic scientists and their industrial peers than observed in the discussed cases.

Acknowledgments This article is based on research conducted within the project "Epistemic Practice, Social Organization, and Scientific Culture: Configurations of Nanoscale Research in Switzerland," funded by the Swiss National Science Foundation (SNSF). For constructive criticism we thank Jörg Potthast, Liliana Doganova, Dominique Vinck, Jochen Gläser, two anonymous reviewers and the participants of the European Workshop "Doing Science-Industry" organized by the PROKNOW consortium at Sofia (September 2008). We are grateful to the observed and interviewed scientists for sharing their time and experience with us. 


\section{References}

Bozeman, Barry. 2000. Technology transfer and public policy: A review of research and theory. Research Policy 29: 627-655.

Carayol, Nicolas. 2003. Objectives, agreements and matching in science-industry collaborations: Reassembling the pieces of the puzzle. Research Policy 32: 887-908.

Ciborra, Claudio U. 1996. The platform organization: Recombining strategies, structures, and surprises. Organization Science 7(2): 103-118.

Etzkowitz, Henry, and Loet Leydesdorff. 2000. The dynamics of innovation: From national systems and "Mode 2" to a Triple Helix of university-industry-government relations. Research Policy 29: $109-123$.

Faulkner, Wendy, and Jacqueline Senker. 1994. Making sense of diversity: Public-private sector research linkage in three technologies. Research Policy 23: 673-695.

Galison, Peter, and Bruce Hevly (eds.). 1992. Big science: The growth of large-scale research. Stanford CA: Stanford University Press.

Geertz, Clifford. 1973. Thick description: Toward an interpretive theory of culture. In: The interpretation of cultures: Selected essays. New York: Basic Books, 3-30.

Gerstein, Marc S. 1992. From machine bureaucracies to networked organizations: An architectural journey. In Organizational architecture, eds. D.A. Nadler, M.S. Gerstein, and R.B. Shaw, 11-38. San Francisco: Jossey-Bass.

Gibbons, Michael, et al. 1994. The new production of knowledge: The dynamics of science and research in contemporary societies. London: Sage.

Gieryn, Thomas F. 2002. What buildings do. Theory and Society 31: 35-74.

Hubert, Matthieu. 2009. Les plates-formes pour la recherche en nanotechnologies: Politiques scientifiques et pratiques de laboratoire à l'épreuve de l'organisation du travail expérimental. Thèse de doctorat. Université de Grenoble.

Hug, Hans Josef. 2007. Welcome to SUL, the Swiss Scanning Probe Microscopy User Laboratory @ Empa. In: Empa (ed.), Swiss Scanning Probe Microscopy User Laboratory (brochure).

Keating, Peter, and Alberto Cambrosio. 2000. Biomedical platforms. Configurations 8: 337-387.

Keating, Peter, and Alberto Cambrosio. 2003. Biomedical platforms: Realigning the normal and the pathological in late-twentieth-century medicine. Cambridge MA, London: MIT Press.

Kim, Dong-Jae, and Bruce Kogut. 1996. Technological platforms and diversification. Organization Science 7(3): 283-301.

Lenoir, Timothy, and Eric Giannella. 2010. Technological platforms and the layers of patent data. In Contexts of invention: Creative production in legal and cultural perspective, eds. Mario Biagioli, Petter Jaszi, and Martha Woodmansee. Chicago: University of Chicago Press.

Merz, Martina. 2010. Reinventing a laboratory: Nanotechnology as a resource for organizational change. In Governing future technologies-Sociology of the sciences 27, eds. Mario Kaiser, et al., 3-19. Dordrecht: Springer.

Meyer-Krahmer, Frieder, and Ulrich Schmoch. 1998. Science-based technologies: University-industry interactions in four fields. Research Policy 27: 835-851.

Mirowski, Philip, and Esther-Mirjam Sent. 2008. The commercialization of science and the response of STS. In The handbook of science and technology studies, eds. Edward J. Hackett, et al., 635-689. Cambridge: MIT Press.

Nowotny, Helga, Peter Scott, and Michael Gibbons. 2001. Re-thinking science: Knowledge and the public in the age of uncertainty. Cambridge UK: Polity.

Owen-Smith, Jason. 2003. From separate systems to a hybrid order: Accumulative advantage across public and private science at Research One universities. Research Policy 32: 1081-1104.

Owen-Smith, Jason. 2005. Trends and transitions in the institutional environment for public and private science. Higher Education 49: 91-117.

Peerbaye, Ashveen, and Vincent Mangematin. 2005. Sharing research facilities: Towards a new mode of technology transfer? Innovation: Management, Policy \& Practice 7: 23-38.

Potthast, Jörg, and Michael Guggenheim. 2008. Are academic spin-offs really doing science? WZB discussion paper SP III 2008-602. Berlin: Wissenschaftszentrum Berlin für Sozialforschung.

Rappert, Brian, Andrew Webster, and David Charles. 1999. Making sense of diversity and reluctance: Academic-industrial relations and intellectual property. Research Policy 28: 873-890. 
Robinson, Douglas K.R., Arie Rip, and Vincent Mangematin. 2007. Technological agglomeration and the emergence of clusters and networks in nanotechnology. Research Policy 36: 871-879.

Shinn, Terry. 1998. The impact of research and education on industry. Industry and Higher Education, October: 270-289.

Shinn, Terry, and Erwan Lamy. 2006. Paths of commercial knowledge: Forms and consequences of university-enterprise synergy in scientist-sponsored firms. Research Policy 35: 1465-1476.

Slaughter, Sheila, and Larry L. Leslie. 1997. Academic capitalism: Politics, policies, and the entrepreneurial university. Baltimore: Johns Hopkins University Press.

Traweek, Sharon. 1992 [1988]. Beamtimes and lifetimes: The world of high energy physicists. Cambridge MA: Harvard University Press.

Tuunainen, Juha, and Tarja Knuuttila. 2009. Intermingling academic and business activities: A new direction for science and universities? Science, Technology, \& Human Values 34(6): 684-704.

Weingart, Peter. 2001. Die Stunde der Wahrheit? Zum Verhältnis der Wissenschaft zu Politik, Wirtschaft und Medien in der Wissensgesellschaft. Weilerswist: Velbrück Wissenschaft. 